\title{
Cognitive Impairment in Juvenile Myoclonic Epilepsy
}

\author{
Sun-Young Kim, M.D. ${ }^{\text {, }}$ Yang-Ha Hwang, M.D. ${ }^{\text {a }}$,Ho-Won Lee, M.D. ${ }^{\text {, }}$,

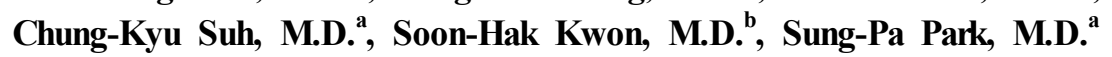 \\ Departments of ${ }^{\mathrm{a}}$ Neurology and ${ }^{\mathrm{b}}$ Pediatrics, School of Medicine, Kyungpook National University, Daegu, Korea
}

\begin{abstract}
Background and Purpose: Cognitive impairments are frequent consequences of epilepsy, with intellectual ability reportedly being lower in patients with idiopathic generalized epilepsies than in the general population. However, neuropsychological investigations have been rarely performed in patients with juvenile myoclonic epilepsy (JME). We aimed to quantify the cognitive function in JME patients using various neuropsychological tests.

Methods: We compared cognitive function in $27 \mathrm{JME}$ patients with that in 27 healthy volunteers using tests examining cognitive performance, such as the verbal and visual memory, frontal function, attention, IQ score, and mood. In the JME group, we examined risk factors for cognitive function such as age, sex, family history, education level, age at seizure onset, seizure frequency, EEG abnormality, disease duration, and previous intake of antiepileptic drugs.

Results: Verbal learning was significantly lower in JME patients than in controls, and attention and verbal fluency were impaired in JME patients compared with controls. However, general intellectual ability and mood did not differ between the groups. Early onset of seizure and long duration of disease were closely related to impaired cognitive function.

Conclusions: JME patients may exhibit impaired cognitive function, in terms of memory and execution, despite having normal intelligence and mood.

J Clin Neurol 3(2):86-92, 2007
\end{abstract}

Key Words : Juvenile myoclonic epilepsy, Cognition, Neuropsychological tests, Mood

\section{INTRODUCTION}

A significant proportion of patients with epilepsy are at an increased risk of cognitive impairment. ${ }^{1,2,10}$ General mental slowness, memory impairment, and attention problems are the most common deficits in epilepsy patients. ${ }^{5}$ Neuropsychology has made a major contribution to characterizing cognition in different types of epilepsy and epileptic syndromes. Most previous investigations have concentrated on determining the localization and lateralization of the epileptogenic abnormality in adult patients with focal epilepsies (mesial temporal lobe epilepsy). ${ }^{20}$ Interest has recently shifted to neuropsychological investigations of nonlesional, so-called idiopathic epilepsies, which have identified specific cognitive and behavioral disturbances that may provide clues concerning the underlying pathogenic process and the potential compensatory mechanisms that are induced. ${ }^{3}$

Juvenile myoclonic epilepsy (JME) is the most important syndrome of idiopathic generalized epilepsy (IGE), and has been associated with frontal lobe dysfunction in recent studies using neuropsychological tests ${ }^{21}$ and various neuroimaging modalities. ${ }^{6-8,27}$ Furthermore, JME is report-

Received : March 30, 2007 / Accepted : May 31, 2007 / Address for correspondence : Sung-Pa Park, M.D.

Department of Neurology, School of Medicine, Kyungpook National University, 50 Samduk 2-ga, Joong-gu, Daegu-si, 700-721, Korea

Tel: +82-53-420-5769, Fax: +82-53-422-4265, E-mail: sppark@mail.knu.ac.kr

* This research was supported by the Kyungpook National University Research Team Fund, 2002. 
edly associated with minimal global cognitive dysfunction, based on neuropsychological tests ${ }^{4}$ and MRI volumetry. ${ }^{9}$ Although two neuropsychological studies have demonstrated the presence of cognitive dysfunction in JME patients, the significance of the results obtained may be diminished by the use of confined neuropsychological tests, epilepsy patients as a control group, medication with antiepileptic drugs (AEDs) before the investigation, and no consideration of psychosocial factors such as mood in the cognitive evaluation. ${ }^{4,21}$

The aim of this study was to quantify cognitive dysfunction in JME patients who did not take AEDs before the investigation. We applied several neuropsychological tests (including mood tests) to JME patients and age-and education-matched healthy subjects.

\section{MATERIALS AND METHODS}

\section{Subjects}

Twenty-seven patients with JME were consecutively enrolled. The inclusion criteria of JME were as follows: juvenile onset of myoclonic jerks on awakening with or without generalized tonic-clonic seizure, generalized discharges of an irregular mixture of 3-6 Hz spiking/ polyspiking slow waves on EEG, no intake of AEDs for at least 6 months prior to the study commencing, older than 16 years, and education minimum of primary school completion. The exclusion criteria were progressive neurological disorders, head injury, mental retardation (IQ score below 70), alcohol or drug abuse, ongoing use of any centrally acting medications, severe psychiatric problems, and other severe medical disorders. We also excluded patients who had a generalized seizure within the previous week or myoclonic jerks within the previous 24 hours.

Twenty-seven age-and education-matched subjects were consecutively collected as a control group during the enrollment of JME patients. The controls had no neurological or psychiatric illness, no family history of seizures, no history of medication, and an IQ score greater than 70 .

\section{Neuropsychological tests}

Neuropsychological tests were administered in a sound-attenuated, temperature-controlled room by a single examiner. According to the literature and our own clinical experience, we selected two cognitive measures that are particularly sensitive to epilepsy patients. ${ }^{25,38} \mathrm{We}$ assessed the memory function through list learning, immediate and delayed word recall, word recognition, and visual reproduction based on the Memory Assessment Scale, which was obtained from Psychological Assessment Resources. ${ }^{11}$ We assessed attention deficit using forward and backward digit spans from the Wechsler Memory Scale-Revised. ${ }^{12}$ We examined attention, visuomotor tracking abilities, and mental flexibility using the Trail-Making Test (TMT) from the HalsteadReitan Battery. ${ }^{13}$ We studied verbal fluency using semantic fluency tests from the Boston Diagnostic Aphasia Examination-Third Edition. ${ }^{14}$ We assessed the general intelligence using a short form of the Korean version of the Wechsler Adult Intelligence Scale. ${ }^{29}$ We also evaluated mood states using the Beck Depression Inventory $(\mathrm{BDI}){ }^{15}$ and Beck Anxiety Inventory (BAI) ${ }^{28}$ Testing sessions lasted about 50 minutes. If a seizure occurred during a neuropsychological examination, testing was suspended and the obtained data were excluded from subsequent analyses.

\section{Study design}

We evaluated differences in cognition, mood states, and intelligence between the JME and control groups. In the JME group, we examined risk factors for cognitive function such as age, sex, family history, education level, age at seizure onset, seizure frequency, EEG abnormality, disease duration, and previous intake of AEDs.

\section{Statistical analysis}

Higher scores indicate better performance on all of the cognitive tests employed except for the TMT (parts A and B), for which higher scores indicate a poorer performance because the time required is defined as the dependent measure in these tests. The data for conti- 
nuous variables are expressed as mean $\pm \mathrm{SD}$ values, and the data for categorized variables are expressed as frequencies. We used the $t$ test for independent samples to compare neuropsychological scores. Fisher's exact test was used for categorized variables, and Pearson's correlation test was used to assess correlations between neuropsychological scores, patient characteristics, and epilepsy variables.

\section{RESULTS}

1. Intergroup differences

The characteristics of patient and controls are listed in Table 1. Age, gender, and education level did not differ significantly between the JME and control groups. The age at seizure onset was $11-15,16-20$, and 21-25 years in 11, 14, and 2 JME patients, respectively. The disease duration was $2.9 \pm 2.1$ years (range, $0.4-9$ years). The proportion of newly diagnosed patient was $81 \%$, and six patients $(22 \%)$ had a family history of epilepsy. The monthly seizure rate was $2.0 \pm 3.0$ (range, $0.3-10$ ). The EEG revealed the presence of spontaneous or photostimulated paroxysmal activity in all patients.

As indicated in Table 2, JME patients exhibit worse performances in list learning $(p<0.01)$, forward and backward digit spans $(p<0.01)$, TMT parts A $(p<0.01)$ and $\mathrm{B}(p<0.05)$, and verbal fluency $(p<0.01)$ compared with those of controls. However, the scores of the IQ test, BAI, and BDI did not differ between the two groups.

\section{Correlation analysis}

The poor performance in neuropsychological tests in the JME group were not correlated with age, sex, family history, education level, seizure frequency, EEG abnormality, or previous intake of AED. However, there were significant correlations between age at seizure onset and verbal fluency $(r=0.481, p<0.05)$ and disease duration and backward digit span ability $(r=0.449$, $p<0.05)$.

\section{DISCUSSION}

We found that intelligence and mood were similar in our JME patients and healthy subjects, whereas the former exhibited specific cognitive deficits such as memory and executive dysfunction. Also, their cognitive function was affected by age at seizure onset and duration of disease.

While intellectual abilities in IGE are usually within the normal range, they tend to be somewhat lower than those in the general population. ${ }^{16,22}$ Behavioral disturbances and poor social adjustment are the most frequently reported problems in IGE. In particular,

Table 1. Characteristics of juvenile myoclonic epilepsy (JME) patients and controls

\begin{tabular}{|c|c|c|c|}
\hline & JME $(n=27)$ & Controls $(n=27)$ & Significance $^{\mathrm{a}}$ \\
\hline Age, years, mean (range) & $19.2(16-29)$ & $20.3(16-27)$ & NS* \\
\hline Sex, male, \% & 67 & 63 & NS \\
\hline \multicolumn{4}{|l|}{ Education, \% } \\
\hline Graduated from primary school & 19 & 11 & NS \\
\hline Graduated from high school & 81 & 89 & NS \\
\hline Age at onset, years, mean (range) & $16.3(12-23)$ & & \\
\hline Duration of epilepsy, years, mean (range) & $2.9(0.4-9)$ & & \\
\hline Newly diagnosed with epilepsy within previous 3 months, \% & 81 & & \\
\hline Family history, \% & 22 & & \\
\hline Seizure rate in previous 3 months, seizures/month, mean (range) & $2.0(0.3-10)$ & & \\
\hline
\end{tabular}

${ }^{a} t$ test for independent samples (two-sided) and Fisher's exact test.

*Nonsignificant (NS), $p>0.05$. 
patients with JME have been found to be somewhat immature, emotionally unstable, and disinhibited, which may result in inadequate social adjustment. ${ }^{17}$ Some of the personality traits and difficulties in social integration that are seen in JME are similar to those observed in patients with frontal lobe lesions. Dysfunction of these regions, especially the prefrontal cortex, results in disturbances in the organization and self-regulation of behavior. ${ }^{18}$ These so-called executive functions are complex cognitive processes that require coordination of several subsystems. ${ }^{34}$ They include the ability to plan and to form abstract concepts, as well as the control of attention, impulsivity, and emotions. ${ }^{3}$ Neuropsychological, histopathological, and neuroimaging studies provide strong evidence for frontal lobe dysfunction in JME. ${ }^{4,6-9,21,27}$ However, studies of other cognitive areas are still scarce. ${ }^{3}$

Two previous neuropsychological studies have demonstrated the cognitive dysfunction in JME patients. ${ }^{4,21}$ Devinsky et al. found a high frequency of impairment of executive functions such as in concept formation, abstract reasoning and mental flexibility, cognitive speed, and planning and organization. ${ }^{21}$ However, that study was limited by the use of a battery of tests that are only sensitive to frontal dysfunction, and by comparing the performance on selected tests with that of patients with temporal lobe epilepsy. Sonmez et al. reported that JME patients had minimal general cognitive dysfunction, impaired verbal and visual memory, worse performance in visuospatial tests, and adverse affects in all frontal lobe tests. ${ }^{4}$ However, the significance of those results may have been decreased by the use of JME patients who were taking valproate. Because adverse effects of AEDs can contribute to cognitive impairment, it is preferable to investigate epilepsy patients who are not receiving AED medications. Another limitation of the study of Sonmez et al. is that since they did not perform mood tests, they were not able to exclude the effects of mood on cognition. Depression, anxiety, and other neuropsychiatric sequelae are known to independently com-

Table 2. Neuropsychological outcomes

\begin{tabular}{|c|c|c|c|}
\hline Measure & $\begin{array}{c}\text { JME }(n=27) \\
\text { mean }(\mathrm{SD})\end{array}$ & $\begin{array}{c}\text { Controls }(n=27) \\
\text { mean }(\mathrm{SD})\end{array}$ & Significance $^{\mathrm{a}}$ \\
\hline List learning & $60.1(7.5)$ & $67.9(3.0)$ & $p<0.01$ \\
\hline Immediate word recall & $11.5(1.0)$ & $11.8(0.5)$ & NS* \\
\hline Delayed word recall & $11.4(1.0)$ & $11.8(0.5)$ & NS \\
\hline Word recognition & $11.9(0.2)$ & $12.0(0.0)$ & NS \\
\hline Visual reproduction & $8.5(1.4)$ & $8.8(1.5)$ & NS \\
\hline Digit span, forward & $9.2(2.7)$ & $10.9(1.5)$ & $p<0.01$ \\
\hline Digit span, backward & $7.4(2.3)$ & $9.9(2.2)$ & $p<0.01$ \\
\hline \multicolumn{4}{|l|}{ Trail-Making Test, part A } \\
\hline Time (sec) & $31.2(10.9)$ & $21.1(5.8)$ & $p<0.01$ \\
\hline No. of errors & $0.2(0.5)$ & $0.0(0.0)$ & NS \\
\hline \multicolumn{4}{|l|}{ Trail-Making Test, part B } \\
\hline Time (sec) & $74.7(27.5)$ & $56.0(25.4)$ & $p<0.05$ \\
\hline No. of errors & $0.5(0.8)$ & $0.1(0.6)$ & NS \\
\hline Verbal fluency & $14.7(3.4)$ & $21.7(6.1)$ & $p<0.01$ \\
\hline IQ score & $97.4(14.6)$ & $103.2(11.0)$ & NS \\
\hline BAI & $7.8(8.8)$ & $7.2(5.8)$ & NS \\
\hline BDI & $8.3(9.6)$ & $7.5(4.8)$ & NS \\
\hline
\end{tabular}

BAI; Beck Anxiety Inventory, BDI; Beck Depression Inventory.

${ }^{\mathrm{a}} t$ test for independent samples (two-sided).

$* p>0.05$.

Higher scores indicate better performance except for the Trail-Making Test. 
promise the integrity of mnestic processing. ${ }^{24}$ Depression can affect the performance of executive functions and the speed of completing a screening test for cognitive function. ${ }^{25}$ Because epilepsy patients can be easily affected by mood disorders, mood changes should be measured simultaneously during a cognitive study so as to discriminate a true cognitive dysfunction from cognitive impairment due to mood changes. In the present study we therefore performed various cognitive tests including mood tests in drug-naive patients, and compared the results with those of healthy subjects.

The cognitive performances of JME patients in the study of Sonmez et al. ${ }^{4}$ were somewhat similar to those of our patients. They found significant impairments of verbal memory and executive function. A recent MRI volumetry study revealed volume changes in the frontal lobe and hippocampus in JME patients, ${ }^{8}$ with the left hippocampus being significantly smaller than the right one. These structural changes might be correlated with the impaired performance on the list-learning test in our cases. Therefore, further studies elucidating memory deficits in JME patients are needed.

Sonmez et al. found that visual memory and visuospatial function were impaired in JME patients, ${ }^{4}$ but this was not the case in our study. These differences might be attributable to Sonmez et al. not performing mood tests, and hence their study not excluding the effect of mood on global cognitive performance. The second explanation is that the duration of the disease may affect the degree of cognition dysfunction. The psychometric intelligence of patients was more severely impaired for a duration of refractory unilateral temporal lobe epilepsy of more than 30 years than for one of 15-30 years. ${ }^{26}$ The longer mean disease duration in the study of Sonmez et al. ${ }^{4}$ (7.2 years) compared with ours (2.9 years) may have resulted in worse cognition in their study. Moreover, the digit span performance was intact in the patients of Sonmez et al. ${ }^{4}$ but impaired in our patients. The digit span test measures attention and short-term memory capacity. Brief disruption of cognitive function as a manifestation of interictal epileptiform discharge (IED) has been well described, ${ }^{35}$ with it being reported in up to $50 \%$ of patients with subclinical epileptiform activity and even in patients during single- spike discharges. These effects can appear during tests of attention, verbal, or nonverbal functions. ${ }^{36}$ IED on EEG was evident in all of our patients, compared with only $57 \%$ of the patients in the study of Sonmez et al. ${ }^{4}$ Therefore, a higher frequency of IED on EEG may be related to an impaired digit span performance.

Some studies have emphasized a decline in the intellectual function of epileptic patients. Lower IQ scores were observed in patients with a younger age at onset, more frequent seizures, more frequent status, longer duration of seizures, structural anomalies of the brain, and atypical absence seizures. ${ }^{16,19}$ However, IQ tests tend not to be sufficiently sensitive to detect subtle neuropsychological changes. ${ }^{37}$ Our study did not reveal any significant differences in IQ score between JME patients and controls despite the presence of abnormal memory and executive functions in the former. A substantial proportion of our patients were newly diagnosed and with a recent occurrence of JME. They did not have severe seizures or concomitant brain pathology, and hence these factors may not affect global intelligence.

The reported overall prevalences of depressive and anxiety disorders in epilepsy patients have been as high as $55 \%$ and $25 \%$, respectively. ${ }^{30}$ Despite the high prevalence of mood disorders in epilepsy, the results of our mood tests did not differ between the JME patients and controls. Known risk factors for depression in epilepsy patients include neurobiological factors, psychosocial factors, and psychotropic effects of AEDs. ${ }^{31}$ Findings of bilateral inferior frontal hypometabolism in patients with partial epilepsy and interictal depression suggest a neurobiologic basis for depression. ${ }^{39}$ However, since our patients had IGE, it is impossible to deduce whether there are neurobiologic risk factors for depression. Psychosocial factors such as stressful life events, poor adjustment to epilepsy, and lower financial status can be a risk factor for depression. ${ }^{32}$ However, since our patients were of school age and had a short duration of disease, psychosocial factors should not have affected depression. Furthermore, since our patients did not take AEDs before the study, psychotropic effects of AEDs were not present. The design of our study - allowing the exclusion of mood affection on cognition - made it possible to demonstrate specific cognitive deficits in 
JME patients.

Cognitive correlates of JME have received little attention despite the extensive studies of cognition in other types of epilepsy. Sonmez et al. found that younger age, presence of a family history, and absence seizures had negative effects on cognitive function, ${ }^{4}$ whereas we demonstrated the harmful effects of early onset of seizure and longer duration of disease. Recent studies have shown that cognitive dysfunction is more frequent in patients with a younger age at seizure onset. ${ }^{23}$ Deterioration of neuropsychological functioning appears to be related to a longer duration of the seizure disorder. ${ }^{26}$ Our findings support these relationships. Unfortunately, the lower incidences of a family history and absence seizures in our cases hindered our ability to demonstrate any relationship between these risks and cognition.

Two questions remain to be answered regarding the relationship between cognition and JME. First, it is difficult to determine whether cognitive and behavioral impairments in JME are caused by stable, disease-related factors or by the acute effects of paroxysmal epileptic activity, such as epileptiform EEG discharges. ${ }^{33}$ Our study demonstrated the harmful effects of an early onset of seizure and longer duration of disease on cognition. These results may be consistent the theory of diseaserelated factors. However, the impaired attention in our patients was probably due to a high frequency of IED, which means that the theory of the acute effects of paroxysmal activity on cognition cannot be ruled out. Neuropsychological studies of patients who are seizurefree after receiving AEDs medication are needed to answer this question. The second unsolved question is whether these impairments are specific to JME or are also applicable to other types of IGE. ${ }^{3}$ Future neuropsychological studies should compare the effects of various idiopathic epileptic syndromes with those of JME.

\section{ACKNOWLEDGMENT}

The authors thank Geum-Ye Bae (a neuropsychologist) for conducting the neuropsychological tests.

\section{REFERENCES}

1. Motamedi G, Meador K. Epilepsy and cognition. Epilepsy Behav 2003;4 Suppl-S38.

2. Kwan P, Brodie MJ. Neuropsychological effects of epilepsy and antiepileptic drugs. Lancet 2001;357:216-222.

3. Hommet C, Sauerwein HC, De Toffol B, Lassonde M. Idiopathic epileptic syndromes and cognition. Neurosci Biobehav Rev 2006;30:85-96.

4. Sonmez F, Atakli D, Sari H, Atay T, Arpaci B. Cognitive function in juvenile myoclonic epilepsy. Epilepsy Behav 2004;5:329-336.

5. Aldenkamp AP. Effect of seizures and epileptiform discharges on cognitive function. Epilepsia 1997;38 Suppl 1:S52-S55.

6. Swartz BE, Simpkins F, Halgren E, Mandelkern M, Brown C, Krisdakumtorn T, et al. Visual working memory in primary generalized epilepsy: an 18FDG-PET study. Neurology 1996;47:1203-1212.

7. Woerman FG, Free SL, Koepp MJ, Sisodiya SM, Duncan JS. Abnormal cerebral structure in juvenile myoclonic epilepsy demonstrated with voxel-based analysis of MRI. Brain 1999;122:2101-2108.

8. Tae WS, Lee EK, Joo EY, Hong SB. Volume changes of frontal lobe and hippocampus in juvenile myoclonic epilepsy. J Korean Neurol Assoc 2003;21:54-61.

9. Tae WS, Joo EY, Hong SB. Distribution of cerebral gray and white matters in juvenile myoclonic epilepsy: voxel based morphometry. J Korean Neurol Assoc 2003;21: 62-69.

10. Cornaggia CM, Beghi M, Provenzi M, Beghi E. Correlation between cognition and behavior in epilepsy. Epilepsia 2006;47 Suppl 2:34-39.

11. Williams JM. Memory Assessment Scales Professional Manual. Psychological Assessment Resources, Odessa, FL, 1991.

12. Wechsler D. Wechsler Memory Scale-Revised Manual. The Psychological Corporation, San Antonio, TX, 1987.

13. Reitan RM, Wolfson D. The Hastead-Reitan Neuropsychological Test Battery: Theory and Clinical Interpretation, 2nd edn. Neuropsychology Press, Tuscon, AZ, 1993.

14. Goodglass H, Kaplan E, Barresi B. Boston Diagnostic Aphasia Examination-Third Edition (BDAE-3). The Psychological Corporation, San Antonio, TX, 2000.

15. Beck AT, Ward CH, Mendelson M, Mock J, Erbaugh J. An inventory for measuring depression. Arch Gen Psychiatry 1961;4:561-571.

16. Farwell JR, Dodrill CB, Batzel LW. Neuropsychological abilities of children with epilepsy. Epilepsia 1985;26:395400 . 
17. Janz D. Epilepsy with impulsive petit mal (juvenile myoclonic epilepsy). Acta Neurol Scand 1985;72:449-459.

18. Damasio H, Grabowski T, Frank R, Galaburda AM, Damasio AR. The return of Phineas Gage: clues about the brain from the skull of a famous patient. Science 1994; 264:1102-1105.

19. Bourgeois BF, Prensky AL, Palkes HS, Talent BK, Busch SG. Intelligence in epilepsy: a prospective study in children. Ann Neurol 1983;14:438-444.

20. Jones-Gotman M, Smith M, Zatorre R. Neuropsychological testing for localizing and lateralizing the epileptogenic region in surgical treatment of the epilepsy. In: Engel J Jr. (ed) Epileptic Seizures and Syndromes. Raven, New York, 1993;245-258.

21. Devinsky O, Gershengorn J, Brown E, Perrine K, Vazquez B, Luciano D. Frontal functions in juvenile myoclonic epilepsy. Neuropsychiatry Neuropsychol Behav Neurol 1997; 10:243-246.

22. Mirsky A, Duncan C, Levav M. Neuropsychology studies in idiopathic generalized epilepsies. In: Jambaque I, Lassonde M, Dulac O (eds) Neuropsychology of Childhood Epilepsy. Plenum, New York, 2001;141-149.

23. Pavone P, Bianchini R, Trifiletti R, Incorpora G, Pavone A, Parano E. Neuropsychological assessment in children with absence epilepsy. Neurology 2001;56:1047-1051.

24. Sapolsky RM. Glucocorticoids and hippocampal atrophy in neuropsychiatric disorders. Arch Gen Psychiatry 2000; 57:925-935.

25. Lutz MT, Helmstaedter C. EpiTrack: tracking cognitive side effects of medication on attention and executive functions in patients with epilepsy. Epilepsy Behav 2005;7: 708-714.

26. Jokeit H, Ebner A. Long term effects of refractory temporal lobe epilepsy on cognitive abilities: a cross sectional study. J Neurol Neurosurg Psychiatry 1999;67: 44-50.

27. Savic I, Lekvall A, Greitz D, Helms G. MR spectroscopy shows reduced frontal lobe concentrations of $\mathrm{N}$-acetyl aspartate in patients with juvenile myoclonic epilepsy.
Epilepsia 2000;41:290-296.

28. Beck AT, Steer RA. Manual for the Beck Anxiety Inventory. The Psychological Corporation, San Antonio, TX, 1990.

29. Kim ZS, Lee YS, Lee MS. Two- and four-subtest short forms of the Korean-Wechsler Adult Intelligence Scale. Seoul J Psychiatry 1994;19:121-126.

30. Jackson MJ, Turkington D. Depression and anxiety in epilepsy. J Neurol Neurosurg Psychiatry 2005;76 Suppl 1:i45-i47.

31. Barry JJ, Lembke A, Gisbert PA, Gilliam F. Affective disorders in epilepsy. In: Ettinger AB, Kanner AM (eds) Psychiatric Issues in Epilepsy: a Practical Guide to Diagnosis and Treatment. Lippincott Williams \& Wilkins, Philadelphia, PA, 2007;203-247.

32. Hermann BP, Whitman S. Neurobiological, psychosocial, and pharmacological factors underlying interictal psychopathology in epilepsy. In: Smith DB, Treiman DM, Trimble MR (eds) Advances in Neurology. Raven, New York, 1991;439-452.

33. Aldenkamp A, Arends J. The relative influence of epileptic EEG discharges, short nonconvulsive seizures, and type of epilepsy on cognitive function. Epilepsia 2004;45: 54-63.

34. Elliott R. Executive functions and their disorders. $\mathrm{Br}$ Med Bull 2003;65:49-59.

35. Meador KJ, Gilliam FG, Kanner AM, Pellock JM. Cognitive and behavioral effects of antiepileptic drugs. Epilepsy Behav 2001;2:SS1-SS17.

36. Binnie CD. Cognitive performance, subtle seizures, and the EEG. Epilepsia 2001;42 Suppl 1:16-18.

37. Loring DW, Meador KJ. Cognitive side effects of antiepileptic drugs in children. Neurology 2004;62:872-877.

38. Lassonde M, Sauerwein HC, Gallagher A, Theriault M, Lepore F. Neuropsychology: traditional and new methods of investigation. Epilepsia 2006;47 Suppl 2:S9-S13.

39. Bromfield EB, Altshuler L, Leiderman DB, Balish M, Ketter TA, Devinsky O, et al. Cerebral metabolism and depression in patients with complex partial seizures. Arch Neurol 1992;49:617-623. 